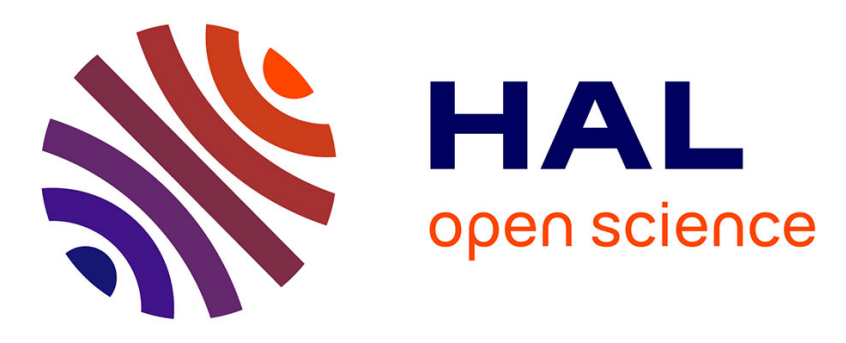

\title{
Multivariate Statistical Techniques for Analog Parametric Test Metrics Estimation
}

Ke Huang, Haralampos-G Stratigopoulos, Abdallah Louay, Salvador Mir, Ahcène Bounceur

\section{- To cite this version:}

Ke Huang, Haralampos-G Stratigopoulos, Abdallah Louay, Salvador Mir, Ahcène Bounceur. Multivariate Statistical Techniques for Analog Parametric Test Metrics Estimation. DTIS 2013, Mar 2013, United Arab Emirates. hal-00780022

\section{HAL Id: hal-00780022 \\ https://hal.univ-brest.fr/hal-00780022}

Submitted on 23 Jan 2013

HAL is a multi-disciplinary open access archive for the deposit and dissemination of scientific research documents, whether they are published or not. The documents may come from teaching and research institutions in France or abroad, or from public or private research centers.
L'archive ouverte pluridisciplinaire $\mathbf{H A L}$, est destinée au dépôt et à la diffusion de documents scientifiques de niveau recherche, publiés ou non, émanant des établissements d'enseignement et de recherche français ou étrangers, des laboratoires publics ou privés. 


\title{
Multivariate Statistical Techniques for Analog Parametric Test Metrics Estimation
}

\author{
Ke Huang ${ }^{\dagger}$, Haralampos-G. Stratigopoulos*, Louay Abdallah*, Salvador Mir*, and Ahcène Bounceur ${ }^{\ddagger}$ \\ ${ }^{\dagger}$ Department of Electrical Engineering, University of Texas at Dallas, Richardson, TX 75080, USA \\ *TIMA Laboratory (CNRS-Grenoble INP-UJF), 46 Av. Félix Viallet, 38031 Grenoble, France

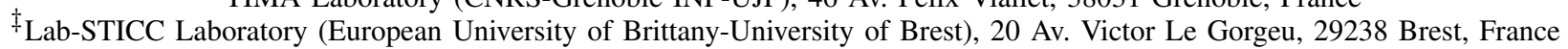

\begin{abstract}
The high cost for testing the analog blocks of a modern chip has sparked research efforts to replace the standard tests with less costly alternative tests. However, test engineers are rather reluctant to adopt alternative tests unless they are evaluated thoroughly before moving to production and they are proven to maintain test quality. This paper gives a comprehensive overview of statistical techniques based on density estimation for evaluating analog parametric test metrics during the test development phase. A large-scale simulation study is carried out for the first time with the aim to demonstrate these techniques in action.
\end{abstract}

Index Terms-Analog, mixed-signal, RF test, test metrics estimation, non-parametric statistics, Copulas, density estimation.

\section{INTRODUCTION}

Consider an analog circuit and let $\mathbf{P}=\left[P_{1}, P_{2}, \cdots, P_{n_{p}}\right]$ be a set of performances that characterize its functionality. The circuit is designed such that $P_{i}$ lies within the desired specification limits $s^{i}=\left(s_{\ell}^{i}, s_{u}^{i}\right), i=1, \cdots, n_{p}$, that is, the performance acceptability region is $A_{P}=\left[s_{\ell}^{1}, s_{u}^{1}\right] \times \cdots \times$ $\left[s_{\ell}^{n_{p}}, s_{u}^{n_{p}}\right]$. There is always a possibility, however, that one or more specifications are violated due to process variations or due to a defect that is induced in one of the fabrication steps. For this reason, every fabricated circuit needs to undergo a test to verify that all the performances are met. The current practice is to perform tests aiming to measure directly the performances. However, these tests incur a high cost which drives up significantly the overall cost of the system wherein the circuit is deployed. Therefore, there is a large incentive to replace these tests with low-cost alternative test measurements $\mathbf{T}=\left[T_{1}, T_{2}, \cdots, T_{n_{t}}\right]$. Let the circuit pass the alternative test $T_{i}$ if $T_{i}$ lies within the test limits $t^{i}=\left(t_{\ell}^{i}, t_{u}^{i}\right), i=1, \cdots, n_{t}$, that is, the test acceptability region is $A_{T}=\left[t_{\ell}^{1}, t_{u}^{1}\right] \times \cdots \times$ $\left[t_{\ell}^{n_{t}}, t_{u}^{n_{t}}\right]$. Then, the question that arises is what the test error is as a result of carrying out alternative tests that do not measure directly the performances, but on which we place test limits. The test error may occur for circuits that contain defects, in which case we refer to it as defect escape, or may occur for circuits with process variations, in which case we refer to it as parametric test error. The focus of this paper is the estimation of parametric test error, which can be broken down into parametric test escape and yield loss, during the test development phase.

Parametric test escape is the probability that a circuit is faulty due to process variations when it has actually been labelled as functional

$$
T_{E}=\operatorname{Pr}\left\{\mathbf{P} \notin A_{P} \mid \mathbf{T} \in A_{T}\right\} .
$$

Yield loss is the probability of labelling a circuit as faulty when it is actually functional

$$
Y_{L}=\operatorname{Pr}\left\{\mathbf{T} \notin A_{T} \mid \mathbf{P} \in A_{P}\right\} .
$$

As is readily seen from (1) and (2), the two parametric test metrics $T_{E}$ and $Y_{L}$ are similar from a statistical point of view. This means that a technique to estimate $T_{E}$ equally applies for the estimation of $Y_{L}$. Notice also that $T_{E}$ and $Y_{L}$ are contradictory objectives. By shrinking the test limits we will achieve a reduction of $T_{E}$, but simultaneously we increase $Y_{L}$, and vice versa, by enlarging the test limits we increase $T_{E}$ and we reduce $Y_{L}$.

The challenge in estimating $T_{E}$ and $Y_{L}$ during the test development phase lies on the fact that their values are typically very small, in the order of a few hundreds parts per million (ppm). Thus, in theory, a very large number of Monte Carlo simulations (e.g. over 1 million) is required so as to estimate $T_{E}$ and $Y_{L}$ with the required precision. Clearly, this simulation effort cannot be afforded during the test development phase, especially when multiple alternative test solutions need to be evaluated before moving to production. Typically, the alternative tests are verified across a subset of fabrication corners and by examining their correlation to the performances for a small Monte Carlo sample. However, this approach entails a risk and may lead to erroneous conclusions. This is perhaps the primary reason why alternative test proposals have not found grounds in industry to date.

The required step to estimate $T_{E}$ and $Y_{L}$ during the test development phase in reasonable time is to speed up circuit simulation. To this end, three techniques based on density estimation have been proposed for the multivariate case [1-3], i.e. when many alternative tests are replacing many standard performance tests, and one technique based on extreme value theory has been proposed for the univariate case [4], i.e. when a single alternative test is replacing a single standard performance test. All techniques provide estimates of test metrics based on a few thousands simulations. The technique in [4] is based on an analytical model, thus the accuracy of the test metrics estimates is guaranteed. In contrast, the techniques in [1-3] tend to be heuristic and the accuracy of the test metrics estimates cannot be fully guaranteed. In [5], the technique in [2] was applied to a case study using test production data and it provided consistent estimates.

In this work, we demonstrate the three techniques in [1$3]$ in action for the first time using a large-scale simulation study. The simulations took up about 3 months to be completed and resulted in a rich data set that allows examining in detail 
the accuracy and limits of these techniques. In particular, we consider an RF low noise amplifier (LNA) and two sensors, namely a current sensor and an envelope detector, that enable a low-cost built-in test. Without loss of generality, we consider only the case of $T_{E}$. Our objective is to estimate the resulting $T_{E}$ when the sensor measurements replace the standard tests for measuring three main performances of the LNA, namely the gain, noise figure, and $S_{11}$. For this purpose, we carried out a Monte Carlo post-layout simulation with 1 million runs. This allowed us to derive the true value of $T_{E}$ with a good accuracy. Thereafter, we employed the three techniques in [13] to obtain fast predictions of $T_{E}$ and we compared these predictions to the true value of $T_{E}$.

The rest of the paper is structured as follows. Next, we discuss in detail the challenge in estimating parametric test metrics. In Section III, we provide brief yet comprehensive descriptions of the techniques in [1-3]. Given that parametric test metrics are statistical quantities, it is more appropriate to report the interval wherein they lie with a certain confidence. Therefore, Section IV discusses the derivation of confidence intervals for statistical quantities. In Section V, we present our case study. In Section VI, we provide the comparison results. Finally, Section VII concludes the paper and points to future work ideas.

\section{Monte Carlo Estimates}

Let $f_{\mathbf{P} \mid \mathbf{T}}\left(\mathbf{P} \mid \mathbf{T} \in A_{T}\right)$ denote the $n_{p}$-dimensional joint probability density function of $\mathbf{P}$ conditional on $\mathbf{T} \in A_{T}$. The test escape can be expressed as

$$
T_{E}=1-\int_{A_{P}} f_{\mathbf{P} \mid \mathbf{T}}\left(\mathbf{P} \mid \mathbf{T} \in A_{T}\right) d \mathbf{P} .
$$

Using the indicator function

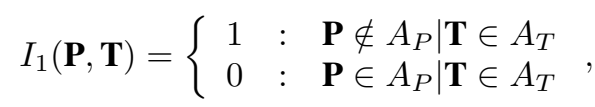

we can write

$$
T_{E}=\int_{\Re^{n}} I_{1}(\mathbf{P}, \mathbf{T}) f_{\mathbf{P} \mid \mathbf{T}}\left(\mathbf{P} \mid \mathbf{T} \in A_{T}\right) d \mathbf{P} .
$$

A straightforward method to approximate the above integral is to perform Monte Carlo circuit-level simulations. For each Monte Carlo run $j$, we record the performances $\mathbf{P}^{j}$ and test measurements $\mathbf{T}^{j}$ and we compute the indicator function $I_{1}\left(\mathbf{P}^{j}, \mathbf{T}^{j}\right)$. An estimate of $T_{E}$ can be obtained using

$$
\hat{T}_{E, M C}=\frac{1}{N} \sum_{j=1}^{N} I_{1}\left(\mathbf{P}^{j}, \mathbf{T}^{j}\right),
$$

where $N$ is the number of runs for which $\mathbf{T} \in A_{T}$.

It is easy to show that $\hat{T}_{E, M C}$ is distributed as $\mathcal{N}\left(T_{E}, \sigma^{2}\right)$ with variance $\sigma^{2}=T_{E}\left(1-T_{E}\right) / N$. Therefore,

$$
\frac{\hat{T}_{E, M C}-T_{E}}{\sigma} \sim \mathcal{N}(0,1)
$$

which gives

$$
\operatorname{Pr}\left\{-z_{\frac{\alpha}{2}} \leq \frac{\hat{T}_{E, M C}-T_{E}}{\sigma} \leq z_{\frac{\alpha}{2}}\right\}=1-\alpha,
$$

where $z_{\frac{\alpha}{2}}$ is the $\left(1-\frac{\alpha}{2}\right)$ quantile of the standard normal distribution, such that $\hat{T}_{E, M C}$ is contained with confidence $100 \cdot(1-\alpha) \%$ within the interval

$$
\left[T_{E}-z_{\frac{\alpha}{2}} \sigma, T_{E}+z_{\frac{\alpha}{2}} \sigma\right] .
$$

If we would like to estimate $T_{E}$ with an accuracy of one order of magnitude less than $T_{E}$, i.e. $z_{\frac{\alpha}{2}} \sigma=0.1 T_{E}$, then the required number of simulations is

$$
N=\left(10 \cdot z_{\frac{\alpha}{2}}\right)^{2} \frac{1-T_{E}}{T_{E}} .
$$

Table I shows the required number of simulations for different confidence levels and values of $T_{E}$. For example, in a typical scenario, assuming a confidence level of $95 \%$ and $T_{E}=100 \mathrm{ppm}, N=3.8 \cdot 10^{6}$. It turns out that the number of simulations required for estimating low values of $T_{E}$ with high confidence is prohibitive. This demonstrates the need for fast statistical simulation methods that can replace efficiently the time-consuming Monte Carlo analysis.

TABLE I

NUMBER OF SIMULATIONS (IN MILLIONS) REQUIRED TO ESTIMATE $T_{E}$ WITH AN ACCURACY OF ONE ORDER OF MAGNITUDE LESS THAN $T_{E}$ AND A CONFIDENCE LEVEL OF $100 \cdot(1-\alpha) \%$.

\begin{tabular}{c|ccccc}
\hline$T_{E}$ & $100 \mathrm{ppm}$ & $200 \mathrm{ppm}$ & $300 \mathrm{ppm}$ & $500 \mathrm{ppm}$ & $1000 \mathrm{ppm}$ \\
\hline $90 \%$ & 2.71 & 1.35 & 0.90 & 0.54 & 0.27 \\
$92 \%$ & 3.06 & 1.53 & 1.02 & 0.61 & 0.31 \\
$95 \%$ & 3.84 & 1.92 & 1.28 & 0.77 & 0.38 \\
$97 \%$ & 5.41 & 2.71 & 1.80 & 1.08 & 0.54 \\
$99 \%$ & 6.63 & 3.32 & 2.21 & 1.33 & 0.66
\end{tabular}

\section{ESTIMATE BASED ON DENSITY ESTIMATION}

Let $\mathbf{X}=[\mathbf{P}, \mathbf{T}]=\left[X_{1}, X_{2}, \cdots, X_{d}\right]$ be the $d$-dimensional random vector that comprises the performances and test measurements, $d=n_{p}+n_{t}$, and let $f_{\mathbf{X}}(\mathbf{x})$ denote the joint probability density function of $\mathbf{X}$. From (1) we can write

$$
T_{E}=\frac{\operatorname{Pr}\left\{\mathbf{P} \notin A_{P}, \mathbf{T} \in A_{T}\right\}}{\operatorname{Pr}\left\{\mathbf{T} \in A_{T}\right\}} .
$$

Using the indicator functions

$$
\begin{gathered}
I_{2}(\mathbf{P}, \mathbf{T})=\left\{\begin{array}{lll}
1 & : & \mathbf{P} \notin A_{P}, \mathbf{T} \in A_{T} \\
0 & : & \text { otherwise }
\end{array}\right. \\
I_{3}(\mathbf{P}, \mathbf{T})=\left\{\begin{array}{lll}
1 & : & \mathbf{T} \in A_{T} \\
0 & : & \text { otherwise }
\end{array}\right.
\end{gathered}
$$

(11) becomes

$$
T_{E}=\frac{\int_{\Re^{d}} I_{2}(\mathbf{P}, \mathbf{T}) f_{\mathbf{X}}(\mathbf{x}) d \mathbf{x}}{\int_{\Re^{d}} I_{3}(\mathbf{P}, \mathbf{T}) f_{\mathbf{X}}(\mathbf{x}) d \mathbf{x}} .
$$

If $\hat{f}_{\mathbf{X}}(\mathbf{x})$ is an estimate of the density $f_{\mathbf{X}}(\mathbf{x})$, then an estimate of $T_{E}$ is obtained as 


$$
\hat{T}_{E, D E}=\frac{\int_{\Re^{d}} I_{2}(\mathbf{P}, \mathbf{T}) \hat{f}_{\mathbf{X}}(\mathbf{x}) d x}{\int_{\Re^{d}} I_{3}(\mathbf{P}, \mathbf{T}) \hat{f}_{\mathbf{X}}(\mathbf{x}) d \mathbf{x}} .
$$

The density estimation approach consists of simulating $N \gg 1$ observations $\mathbf{X}^{j}=\left[\mathbf{P}^{j}, \mathbf{T}^{j}\right]$ of $\mathbf{X}$ from the density $\hat{f}_{\mathbf{X}}(\mathbf{x})$ and calculating the indicator functions $I_{2}$ and $I_{3}$ on each observation. Then, an estimate of $T_{E}$ is obtained as

$$
\hat{T}_{E, D E}=\frac{\sum_{j=1}^{N} I_{2}\left(\mathbf{P}^{j}, \mathbf{T}^{j}\right)}{\sum_{j=1}^{N} I_{3}\left(\mathbf{P}^{j}, \mathbf{T}^{j}\right)} .
$$

\section{A. Multinormal density [1]}

We assume that $f_{\mathbf{X}}(\mathbf{x})$ is Gaussian with $d \times 1$ mean vector $\mu$ and $d \times d$ covariance matrix $\Sigma$, i.e.

$$
\hat{f}_{\mathbf{X}}(\mathbf{x})=\frac{1}{(2 \pi)^{\frac{1}{d}}|\Sigma|^{\frac{1}{2}}} e^{-\frac{1}{2}(\mathbf{x}-\mu)^{T} \Sigma^{-1}(\mathbf{x}-\mu)} .
$$

The mean vector and the covariance matrix are estimated based on data from an initial Monte Carlo circuit-level simulation with $n$ runs that we can afford. A new sample from $\hat{f}_{\mathbf{X}}(\mathbf{x})$ can be generated as

$$
\mathbf{X}=\mu+A \mathbf{W},
$$

where $A$ is the Cholesky decomposition of the covariance matrix $\Sigma$ and $\mathbf{W}$ is a $d \times 1$ vector whose components are independent random samples of the univariate standard normal distribution.

\section{B. Non-parametric density [2]}

This approach revokes the normality hypothesis and can be applied regardless of the parametric form of $f_{\mathbf{X}}(\mathbf{x})$, i.e. even when the marginal distributions of $f_{\mathbf{X}}(\mathbf{x})$ have distinct parametric forms resulting in an undocumented form for $f_{\mathbf{X}}(\mathbf{x})$. Let $\mathbf{X}^{j}, j=1, \ldots, n$, be the data from an initial Monte Carlo circuit-level simulation with $n$ runs that we can afford. The data are pre-scaled such that in each coordinate we have the same spread. For the purpose of simplicity, however, we do not change the notation. The inverse transformation can be applied any time to return to the original space. The nonparametric kernel density estimate of $f_{\mathbf{X}}(\mathbf{x})$ is given by [6]

$$
\hat{f}_{\mathbf{X}}(\mathbf{x}, \alpha)=\frac{1}{n} \sum_{j=1}^{n} \frac{1}{\left(\lambda_{j}(\alpha) \cdot h\right)^{d}} K\left(\frac{\mathbf{x}-\mathbf{X}^{j}}{\lambda_{j}(\alpha) \cdot h}\right),
$$

where

$$
h=\left\{8 c_{d}^{-1}(d+4)(2 \sqrt{\pi})^{d}\right\}^{1 /(d+4)} n^{-1 /(d+4)}
$$

is a smoothing parameter called bandwidth,

$$
c_{d}=2 \pi^{d / 2} /(d \cdot \Gamma(d / 2))
$$

is the volume of the unit $d$-dimensional sphere,

$$
K_{e}(\mathbf{t})= \begin{cases}\frac{1}{2} c_{d}^{-1}(d+2)\left(1-\mathbf{t}^{T} \mathbf{t}\right) & \text { if } \mathbf{t}^{T} \mathbf{t}<1 \\ 0 & \text { otherwise }\end{cases}
$$

is the Epanechnikov kernel, $\lambda_{j}$ are local bandwidth factors defined by

$$
\lambda_{j}(\alpha)=\left\{\hat{f}_{\mathbf{X}}\left(\mathbf{X}^{j}, 0\right) / g\right\}^{-\alpha},
$$

and $g$ is the geometric mean

$$
\log g=n^{-1} \sum_{j=1}^{n} \log \hat{f}_{\mathbf{X}}\left(\mathbf{X}^{j}, 0\right) .
$$

The density estimate in (19) is a weighted sum of kernels centered on the $n$ observations. The bandwidth $h$ defines the width of the kernels. The parameter $\lambda_{j}(\alpha)$ multiplies the bandwidth of the kernel of the $j$-th observation. By increasing $\alpha$, the tails of the density estimate become smoother and longer, but less heavier [6]. Practically, by increasing $\alpha$ we increase the probability of sampling an "extreme" vector $\mathbf{X}$. It can be shown that $\hat{f}_{\mathbf{X}}(\mathbf{x}, \alpha) \rightarrow f_{\mathbf{X}}(\mathbf{x})$ in probability as $n \rightarrow \infty$ provided that the selected bandwidth satisfies $h \rightarrow 0$ and $n h \rightarrow \infty$ as $n \rightarrow \infty$ [6]. The choice of the bandwidth in (20) is made following an approach known as rule-of-thumb [6] and satisfies these conditions. The default value of $\alpha$ often adopted by practitioners is $\alpha=0$, resulting in $\lambda_{j}(0)=1$ for all $n$ observations.

A new sample $\mathbf{X}$ can be generated as follows:

Step 1 Consider an observation $\mathbf{X}^{I}$ with $I$ chosen from $\{1, \ldots, n\}$ uniformly at random.

Step 2 Generate $\mathbf{v}$ to have probability density function $K_{e}(\mathbf{v})$ in (22).

Step 3 Set $\mathbf{X}=\mathbf{X}^{I}+h \lambda_{I}(\alpha) \mathbf{v}$.

The acceptance-rejection method is used in Step 2, in order to simulate from the kernel estimate $K_{e}$. The method relies on identifying a density function $f_{0}$ that can be (a) simulated much easier and (b) scaled with some constant $c$ so that it majorizes $K_{e}$, that is, so that $K_{e}(\mathbf{v}) \leq c \cdot f_{0}(\mathbf{v}), \forall \mathbf{v} \in R^{d}$. The method can be visualized as choosing a subsequence from an independent identically distributed sequence drawn from $f_{0}$, in such a way that the subsequence has probability density function $K_{e}$ :

Step $2 a$ Generate $\mathbf{v}$ to have probability density function $f_{0}$.

Step $2 b$ Generate $u$ from a uniform distribution in $[0,1]$.

Step $2 c$ If $u \leq K_{e}(\mathbf{v}) /\left(c \cdot f_{0}(\mathbf{v})\right)$ accept and return $\mathbf{v}$, otherwise return to step 2a.

In the case of the Epanechnikov kernel, we can select $f_{0}$ to be the uniform distribution in $[-1,1]^{n+d}$ and $c=c_{n+d}^{-1}(n+$ $d+2) / 2$.

\section{Gaussian copula [3]}

Let $F_{i}\left(x_{i}\right) \equiv \operatorname{Pr}\left\{X_{i} \leq x_{i}\right\}$ denote the distribution function of $X_{i}, i=1, \cdots, d$. This approach uses the transformations $U_{i}=F_{i}\left(X_{i}\right)$ to map $\mathbf{X}$ to $\mathbf{U}=\left[U_{1}, U_{2}, \cdots, U_{d}\right]$, where $\mathbf{U} \in[0,1]^{d}$. The distribution function of $\mathbf{U}$ is called the copula of $\mathbf{X}$. If the distribution $F(\mathbf{x})$ of $\mathbf{X}$ is Gaussian, then the resulting copula is called the Gaussian copula. The key observation is that even if $F(\mathbf{x})$ is not Gaussian, then it is possible that the resulting copula is Gaussian. Under the assumption that the resulting copula is Gaussian, we can apply 
the following procedure to generate $N \gg 1$ observations of X. Apply the transformations $Y_{i}=\Phi^{-1}\left(U_{i}\right), i=1, \cdots, d$, to map $\mathbf{U}$ to $\mathbf{Y}=\left[Y_{1}, Y_{2}, \cdots, Y_{d}\right]$, where $\Phi$ denotes the standard Gaussian distribution function. Then, by definition, the density $f_{\mathbf{Y}}(\mathbf{y})$ of $\mathbf{Y}$ is Gaussian. Therefore, we can fit a Gaussian density to the observations $\mathbf{Y}=\left[\mathbf{Y}^{1}, \mathbf{Y}^{2}, \cdots, \mathbf{Y}^{n}\right]$ and sample this density to obtain $N \gg 1$ observations of $\mathbf{Y}$, as discussed in Section III-A. Then we apply the inverse transformation $X_{i}=F_{i}^{-1}\left(\Phi\left(Y_{i}\right)\right)$ to obtain $N \gg 1$ observations of $\mathbf{X}$.

\section{STATISTICS AND CONFIDENCE INTERVALS}

\section{A. Classical approach}

Let $\mathbf{X}=\left[X_{1}, X_{2}, \cdots, X_{d}\right]$ be a $d$-dimensional random vector and let $\rho(\mathbf{X})$ be some interesting quantity of $\mathbf{X}$ with mean $\mu_{\rho}$ and variance $\sigma_{\rho}^{2}$. The simplest way to estimate $\mu_{\rho}$ and $\sigma_{\rho}^{2}$ is to generate $N$ samples of size $n$. In particular, let $Z^{i}=\left\{\mathbf{X}^{1 i}, \mathbf{X}^{2 i}, \cdots, \mathbf{X}^{n i}\right\}, i=1, \cdots, N$, denote the $i$ th sample, where $\mathbf{X}^{j i}$ is the $j$-th observation of $\mathbf{X}$ in the $i$-th sample, and let $\rho\left(Z^{i}\right)$ denote the value of $\rho$ for the $i$-th sample. Then, natural estimators for $\mu_{\rho}$ and $\sigma_{\rho}^{2}$ are the sample mean $\bar{\rho}$ and sample variance $s_{\rho}^{2}$

$$
\begin{gathered}
\bar{\rho}=\frac{1}{N} \sum_{i=1}^{N} \rho\left(Z^{i}\right), \\
s_{\rho}^{2}=\frac{1}{N-1} \sum_{i=1}^{N}\left(\rho\left(Z^{i}\right)-\bar{\rho}\right)^{2} .
\end{gathered}
$$

Furthermore, by the central limit theorem [7], for large $N$,

$$
\frac{\sqrt{N}\left(\bar{\rho}-\mu_{\rho}\right)}{\sigma_{\rho}} \sim \mathcal{N}(0,1) .
$$

Thus,

$$
\operatorname{Pr}\left\{-z_{\frac{\alpha}{2}} \leq \frac{\sqrt{N}\left(\bar{\rho}-\mu_{\rho}\right)}{\sigma_{\rho}} \leq z_{\frac{\alpha}{2}}\right\}=1-\alpha .
$$

Rearranging (28) gives

$$
\operatorname{Pr}\left\{\bar{\rho}-z_{\frac{\alpha}{2}} \sigma_{\rho} / \sqrt{N} \leq \mu_{\rho} \leq \bar{\rho}+z_{\frac{\alpha}{2}} \sigma_{\rho} / \sqrt{N}\right\}=1-\alpha
$$

such that

$$
\left[\bar{\rho}-z_{\frac{\alpha}{2}} \sigma_{\rho} / \sqrt{N}, \bar{\rho}+z_{\frac{\alpha}{2}} \sigma_{\rho} / \sqrt{N}\right]
$$

is the $100 \cdot(1-\alpha) \%$ confidence interval for $\mu_{\rho}$. Replacing $\sigma_{\rho}$ by $s_{\rho}$ leads to

$$
\left[\bar{\rho}-z_{\frac{\alpha}{2}} s_{\rho} / \sqrt{N}, \bar{\rho}+z_{\frac{\alpha}{2}} s_{\rho} / \sqrt{N}\right]
$$

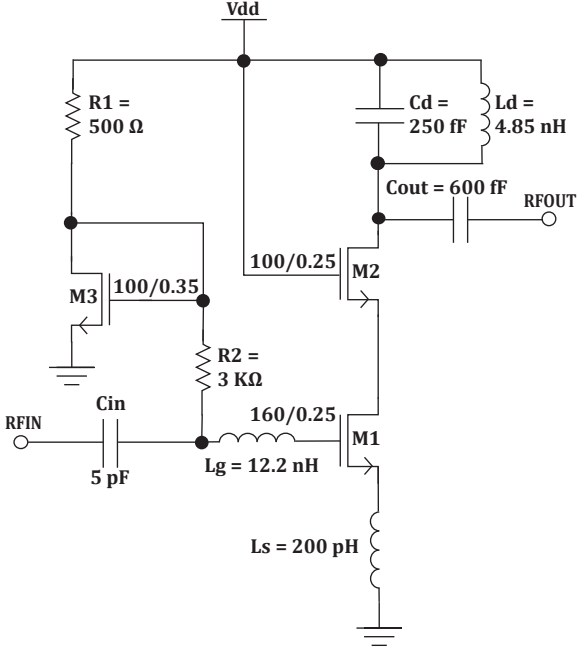

Fig. 1. CMOS low noise amplifier (LNA).

\section{B. Bootstrap}

The bootstrap technique offers a general approach to estimate the statistics of $\rho(\mathbf{X})$ based on a single sample of size $n$, denoted by $Z=\left\{\mathbf{X}^{1}, \mathbf{X}^{2}, \cdots, \mathbf{X}^{n}\right\}$. It is particularly appealing in the case where it requires a large computational effort to collect $N \times n$ observations of $\mathbf{X}$, in order to use (25) and (26). The basic idea is to generate $B$ samples of size $n$ by successively selecting uniformly with replacement from $Z$. In particular, let $Z^{* i}=\left\{\mathbf{X}^{* 1 i}, \mathbf{X}^{* 2 i}, \cdots, \mathbf{X}^{* n i}\right\}, i=1, \cdots, B$, denote the $i$-th bootstrap sample and let $\rho\left(Z^{* i}\right)$ denote the value of $\rho$ for the $i$-th bootstrap sample. Then, the bootstrap estimators for $\mu_{\rho}$ and $\sigma_{\rho}^{2}$ are

$$
\begin{gathered}
\hat{\mu}_{\rho}=\frac{1}{B} \sum_{i=1}^{B} \rho\left(Z^{* i}\right) \\
\hat{\sigma}_{\rho}^{2}=\frac{1}{B-1} \sum_{i=1}^{B}\left(\rho\left(Z^{* i}\right)-\hat{\mu}_{\rho}\right)^{2} .
\end{gathered}
$$

Assuming that the distribution of $\rho\left(Z^{*}\right)$ is symmetrical and reveals no bias, that is, it is centered on $\mu_{\rho}$, then the $100 \cdot(1-$ $\alpha) \%$ confidence interval for $\mu_{\rho}$ is given by

$$
\left[\hat{\mu}_{\rho}-z_{\frac{\alpha}{2}} \hat{\sigma}_{\rho} / \sqrt{B}, \hat{\mu}_{\rho}+z_{\frac{\alpha}{2}} \hat{\sigma}_{\rho} / \sqrt{B}\right]
$$

\section{CAse Study}

Our test vehicle is an inductive source-degenerated cascode LNA used in the $802.11 \mathrm{~g}$ standard receivers that operate in the $2.4 \mathrm{GHz}$ ISM band. The schematic of the LNA is shown in Fig. 1. It is designed using the $0.25 \mu \mathrm{m}$ Qubic4+ technology by NXP Semiconductors. In this case study, we are investigating whether it is possible to replace the standard tests for measuring gain, NF, and $S_{11}$ by two built-in tests that employ an envelope detector (ED) and a current sensor (CS). In the test mode, the LNA is stimulated with a $2.4 \mathrm{GHz}$ sinusoidal of amplitude $-30 \mathrm{dBm}$. The ED, shown in Fig. 2, measures the RMS value of the LNA's RF output. The CS, shown in Fig. 3, measures the dynamic power supply current 


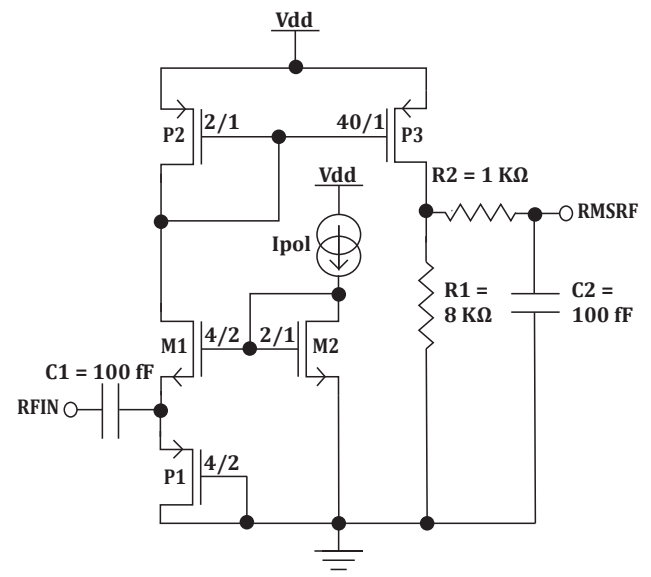

Fig. 2. CMOS envelope detector.

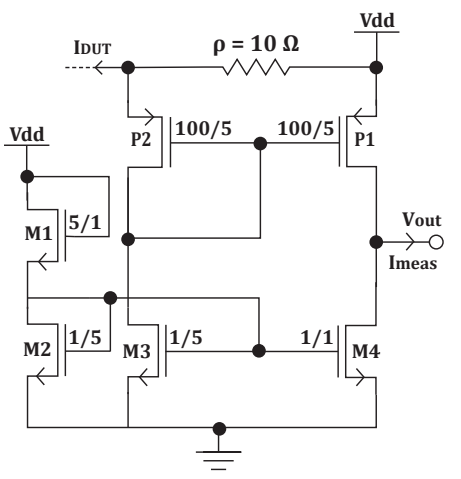

Fig. 3. CMOS built-in current sensor.

flowing through the LNA. Its operation is based on monitoring the voltage drop across the small parasitic resistor $\rho$ between the power supply pad and the core of the LNA. First, we record the output of the ED, then the input of the ED is switched to the output of the CS, in order to record the RMS value of the power supply current. The built-in test approach using envelope detectors and current sensors has been extensively studied in the literature [8-12]. It is cost-effective since only DC signals carrying RF information are extracted off-chip.

The specifications of the three performances are set at $k_{1} \cdot \sigma$, i.e.

$$
\begin{aligned}
\text { gain } & \geq s_{\text {gain }}=\mu_{\text {gain }}-k_{1} \cdot \sigma_{\text {gain }} \\
\mathrm{NF} & \leq s_{\mathrm{NF}}=\mu_{\mathrm{NF}}+k_{1} \cdot \sigma_{\mathrm{NF}} \\
S_{11} & \leq s_{S_{11}}=\mu_{S_{11}}+k_{1} \cdot \sigma_{S_{11}},
\end{aligned}
$$

where the means and standard deviations are computed on an initial small Monte Carlo sample and $k_{1}$ is a multiplication coefficient. From simulations, we observed that the DC measurements provided by the ED and CS, denoted respectively by $T_{E D}$ and $T_{C S}$, are proportional to gain and inversely proportional to $N F$ and $S_{11}$. Thus, we place lower test limits on $T_{E D}$ and $T_{C S}$ at $k_{2} \cdot \sigma$, i.e.

$$
\begin{aligned}
& T_{E D} \geq t_{T_{E D}}=\mu_{T_{E D}}-k_{2} \cdot \sigma_{T_{E D}} \\
& T_{C S} \geq t_{T_{C S}}=\mu_{T_{C S}}-k_{2} \cdot \sigma_{T_{C S}},
\end{aligned}
$$

where as before the means and standard deviations are computed on an initial small Monte Carlo sample and $k_{2}$ is a multiplication coefficient. Therefore, the parametric test escape is expressed as

$$
\begin{aligned}
T_{E}= & \operatorname{Pr}\left\{\text { gain }<s_{\text {gain }} \cup \mathrm{NF}>s_{\mathrm{NF}} \cup \cdots\right. \\
& \left.\cup S_{11}>s_{S_{11}} \mid T_{E D} \geq t_{T_{E D}}, T_{C S} \geq t_{T_{C S}}\right\} .
\end{aligned}
$$

\section{RESULTS}

We carried out a post-layout Monte Carlo simulation analysis of the LNA with the embedded sensors. We generated in total $10^{6}$ samples which took up about 3 months. For each sample, we recorded the performances and test measurements, that is, the values of $\mathbf{X}=$ [gain, NF, $\left.S_{11}, T_{E D}, T_{C S}\right]$. Using (6), we obtained an estimate of $T_{E}$ which is close to the true value. Next, we considered a random set of $n=5 \cdot 10^{3}$ samples out of the available $10^{6}$ and we used the three techniques discussed in Section III, in order to generate $N=10^{6}$ observations of $\mathbf{X}$ corresponding to $10^{6}$ instances of the LNA. These data are used to obtain estimates of $T_{E}$ using (16). Notice that obtaining $10^{6}$ instances of the LNA using any of the techniques in Section III takes up a few minutes. The fast estimates of $T_{E}$ are compared to the true value of $T_{E}$ that is obtained using the time-consuming Monte Carlo experiment.

Table II shows the $95 \%$ confidence intervals of $T_{E}$ based on 10 bootstrap samples using different estimation techniques, namely the time-consuming $\mathrm{MC}$, the multinormal density, the non-parametric density using two different values for $\alpha$, and the Gaussian copula. The specifications are set at $k_{1}=4$ sigma while the test limits are set at $k_{2}$ sigma with $k_{2}=\{1,2,3,4\}$. Fig. 4 plots Table II. Table III and Fig. 5 show the respective results for $k_{1}=5$. The following observations can be made:

1) As shown by the "reference" MC curve, as $k_{2}$ increases, the test becomes less strict and, thereby, $T_{E}$ increases.

2) The techniques based on multinormal density and Gaussian copula underestimate the $T_{E}$ for certain values of $k_{2}$. The reason is that the underlying assumptions for these techniques are not satisfied. In particular, NF and $S_{11}$ turn out to follow a generalized extreme value (GEV) distribution while gain, $T_{E D}$, and $T_{C S}$ turn out to follow a Gaussian distribution. As a result, the joint distribution $f_{\mathbf{X}}(\mathbf{x})$ is not Gaussian. The resulting copula is not Gaussian either. It turns out to be a mixed copula where most pairs of performances and tests have a Gaussian copula, but others appear to have a Gumbel copula, i.e. a copula resulting from a Gumbel bivariate distribution. The theory for mixed copulas is not well developed yet. Notice that the multinormal density and Gaussian copula techniques should be used only if their assumptions are met, otherwise their utilization entails a risk. Nevetheless, we used them in our case study with the aim to evaluate the prediction errors that we commit.

3) The non-parametric density technique with the default value $\alpha=0$ provides estimates that track well the increase of $T_{E}$ with $k_{2}$. The confidence intervals of the estimates overlap with those of the MC except in the case of $k_{2}=4$ : for $k_{1}=4$ the $T_{E}$ is overestimated by about $150 \mathrm{ppm}$ while for $k_{1}=5$ the $T_{E}$ is underestimated by about $10 \mathrm{ppm}$. There are two 
TABLE II

$T_{E}$ 95\% CONFIDENCE INTERVALS USING DIFFERENT ESTIMATION TECHNIQUES FOR $k_{1}=4$.

\begin{tabular}{|c||c||c||c||c||c|}
\hline$k_{2}$ & $\begin{array}{c}\text { MC } \\
\left(10^{6}\right)\end{array}$ & $\begin{array}{c}\text { Multinormal } \\
\left(5 \cdot 10^{3}\right)\end{array}$ & $\begin{array}{c}\text { Non-param. } \\
\alpha=0 \\
\left(5 \cdot 10^{3}\right)\end{array}$ & $\begin{array}{c}\text { Non-param. } \\
\alpha=-0.1 \\
\left(5 \cdot 10^{3}\right)\end{array}$ & $\begin{array}{c}\text { Copulas } \\
\left(5 \cdot 10^{3}\right)\end{array}$ \\
\hline 1 & {$[0,1]$} & {$[10,14]$} & {$[0,0]$} & {$[0,0]$} & {$[0,0]$} \\
\hline 2 & {$[27,33]$} & {$[27,33]$} & {$[31,39]$} & {$[17,24]$} & {$[8,11]$} \\
\hline 3 & {$[278,311]$} & {$[52,65]$} & {$[311,332]$} & {$[265,285]$} & {$[52,62]$} \\
\hline 4 & {$[377,400]$} & {$[86,93]$} & {$[536,584]$} & {$[467,487]$} & {$[106,115]$} \\
\hline
\end{tabular}

TABLE III

$T_{E} 95 \%$ CONFIDENCE INTERVALS USING DIFFERENT ESTIMATION TECHNIQUES FOR $k_{1}=5$.

\begin{tabular}{|c||c||c||c||c||c|}
\hline$k_{2}$ & $\begin{array}{c}\text { MC } \\
\left(10^{6}\right)\end{array}$ & $\begin{array}{c}\text { Multinormal } \\
\left(5 \cdot 10^{3}\right)\end{array}$ & $\begin{array}{c}\text { Non-param. } \\
\alpha=0 \\
\left(5 \cdot 10^{3}\right)\end{array}$ & $\begin{array}{c}\text { Non-param. } \\
\alpha=0.1 \\
\left(5 \cdot 10^{3}\right)\end{array}$ & $\begin{array}{c}\text { Copulas } \\
\left(5 \cdot 10^{3}\right)\end{array}$ \\
\hline 1 & {$[0,0]$} & {$[0,0]$} & {$[0,0]$} & {$[0,0]$} & {$[0,0]$} \\
\hline 2 & {$[0,0]$} & {$[0,0]$} & {$[1,1]$} & {$[0,2]$} & {$[0,0]$} \\
\hline 3 & {$[10,16]$} & {$[0,0]$} & {$[9,13]$} & {$[13,16]$} & {$[0,0]$} \\
\hline 4 & {$[27,34]$} & {$[0,1]$} & {$[15,20]$} & {$[20,27]$} & {$[0,0]$} \\
\hline
\end{tabular}

reasons for this small disagreement. First, the convergence of the non-parametric density in (19) to the true density $f_{\mathbf{X}}(\mathbf{x})$ is not guaranteed for a small initial sample of size $n$. Second, there exist no technique to chose optimally the values of $h$ and $\alpha$ such that the convergence is expedited. For $k_{1}=4$, a better choice would have been to choose an $\alpha$ lower than zero, in order to have shorter tails and, thereby, less $T_{E}$. Following the same argument, for $k_{1}=5$, a better choice would have been to choose an $\alpha$ larger than zero. As shown in Tables II and III and in Fig. 4 and 5, these choices improve the results for $k_{2}=4$.

\section{CONCLUSION}

We carried out for the first time a time-consuming Monte Carlo experiment to examine the accuracy of three test metrics estimation techniques previously reported in the literature. The multinormal technique should be used provided that the underlying distribution is Gaussian. If not, then the Gaussian copula technique should be used provided that the underlying copula is Gaussian. If not, then the last resort is to use the general non-parametric technique. For our case study, the assumptions of the multinormal and Gaussian copula techniques are not met while the non-parametric technique provided good estimates with ppm precision. Future work will focus on generalizing the univariate technique in [4] to many dimensions, on extending the Gaussian copula technique to mixed copulas, and on generating more case studies for comparison purposes.

\section{REFERENCES}

[1] A. Bounceur, S. Mir, E. Simeu, and L. Rolindez, "Estimation of test metrics for the optimisation of analogue circuit testing," Journal of Electronic Testing: Theory and Applications, vol. 23, no. 6, pp. 471-484, 2007.

[2] H.-G. Stratigopoulos, S. Mir, and A. Bounceur, "Evaluation of analog/RF test measurements at the design stage," IEEE Transactions on ComputerAided Design of Integrated Circuits and Systems, vol. 28, no. 4, pp. 582590, 2009.

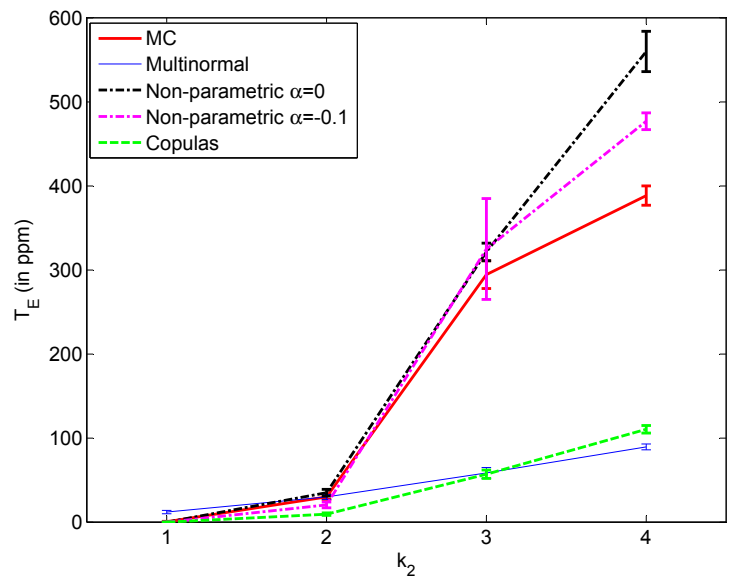

Fig. 4. Performance of test metrics estimation methods for $k_{1}=4$.

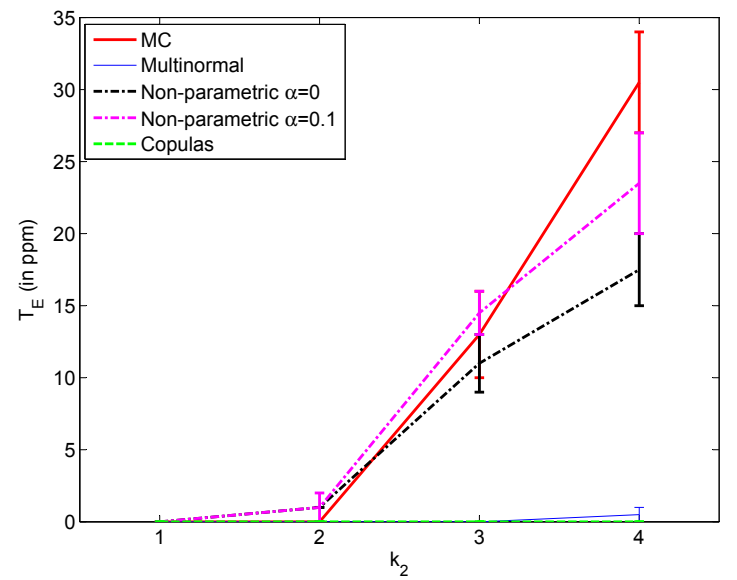

Fig. 5. Performance of test metrics estimation methods for $k_{1}=5$.

[3] A. Bounceur, S. Mir, and H.-G. Stratigopoulos, "Estimation of analog parametric test metrics using copulas," IEEE Transactions on ComputerAided Design of Integrated Circuits and Systems, vol. 30, no. 9, pp. 14001410, 2011.

[4] H.-G. Stratigopoulos and S. Mir, "Analog test metrics estimates with PPM accuracy," in Proc. IEEE/ACM International Conference on ComputerAided Design, 2010, pp. 241-247.

[5] N. Kupp, H.-G. Stratigopoulos, Y. Makris, and P. Drineas, "On proving the efficiency of alternative RF tests," in Proc. IEEE/ACM International Conference on Computer-Aided Design, 2011, pp. 762-767.

[6] B. W. Silverman, Density Estimation for Statistics and Data Analysis, Chapman \& Hall/CRC, 1986.

[7] A. Papoulis, Probability, Random Variables, and Stochastic Processes, McGraw-Hill, 3rd edition, 1991.

[8] A. Gopalan, M. Margala, and P. R. Mukund, "A current based self-test methodology for RF front-end circuits," Microelectronics Journal, vol. 36, no. 12, pp. 1091-1102, 2005.

[9] A. Valdes-Garcia, R. Venkatasubramanian, J. Silva-Martinez, and E. Sanchez-Sinencio, "A broadband CMOS amplitude detector for onchip RF measurements," IEEE Transactions on Instrumentation and Measurement, vol. 57, no. 7, pp. 1470-1477, 2008.

[10] Y.-C. Huang, H.-H. Hsieh, and L.-H. Lu, "A built-in self-test technique for RF low-noise amplifiers," IEEE Transactions on Microwave Theory and Techniques, vol. 56, no. 2, pp. 1035-1042, 2008.

[11] M. Cimino, H. Lapuyade, Y. Deval, T. Taris, and J.-B. Bégueret, "Design of a $0.9 \mathrm{~V} 2.45 \mathrm{GHz}$ self-testable and reliability-enhanced CMOS LNA," IEEE Journal of Solid-State Circuits, vol. 43, no. 5, pp. 1187-1194, 2008.

[12] L. Abdallah, H.-G. Stratigopoulos, S. Mir, and C. Kelma, "RF front-end test using built-in sensors," IEEE Design \& Test of Computers, vol. 28, no. 6 , pp. $76-84,2011$. 